\title{
Quelles sont les causes de la déforestation dans le bassin du Congo ? Synthèse bibliographique et études de cas
}

\author{
Pauline Gillet ${ }^{(1)}$, Cédric Vermeulen ${ }^{(1)}$, Laurène Feintrenie ${ }^{(2)}$, Hélène Dessard ${ }^{(3)}$, \\ Claude Garcia ${ }^{(4)}$
}

\author{
(1) Université de Liège - Gembloux Agro-Bio Tech. Biosystems Engineering. Passage des Déportés, 2. BE-5030 Gembloux \\ (Belgique).E-mail : pgillet@ulg.ac.be \\ (2) CIRAD. Direction Régionale d'Afrique Centrale. Unité de Recherche Biens et Services des Écosystèmes Forestiers \\ tropicaux (UR B\&SEF). Département Environnement et Sociétés. BP 2572. Rue Joseph Essono Balla. Yaoundé (Cameroun). \\ (3) CIRAD. Unité de Recherche Biens et Services des Écosystèmes Forestiers tropicaux (UR B\&SEF). Département \\ Environnement et Sociétés. Campus international de Baillarguet. TA C-105/D. Montpellier cedex 5 (France). \\ (4) ETH Zurich. Unité de Recherche Biens et Services des Écosystèmes Forestiers tropicaux (UR B\&SEF). FORDEV. \\ Department of Environmental Systems Science. Département Environnement et Sociétés. Zurich 8092 (Switzerland).
}

Reçu le 20 mai 2015, accepté le 11 avril 2016.

\begin{abstract}
Description du sujet. Les forêts du Bassin du Congo font partie des zones forestières les mieux préservées de la planète. Néanmoins, les facteurs qui entrainent la déforestation ailleurs dans le monde se manifestent également dans cette sous-région du globe. Cet article propose une revue de la littérature des causes directes et des facteurs sous-jacents de la destruction du couvert forestier des régions tropicales, afin de mettre en exergue les moteurs de la déforestation dans le bassin du Congo, et plus particulièrement au Cameroun et au Gabon.
\end{abstract}

Littérature. Les causes directes de déforestation, définies comme ayant un lien cause-conséquence immédiat avec la destruction du couvert forestier, sont renforcées par des facteurs sous-jacents tels que les facteurs économiques, les avancées technologiques, les mesures politiques ainsi que les pressions démographiques. Toutes ces causes interagissent de façons distinctes dans les différentes régions tropicales de par le monde et expliquent les divergences et similitudes entre les dynamiques de déforestation régionales. En plus de l'expansion de l'infrastructure, du développement du secteur minier et de l'extraction du bois, l'agriculture représente la cause directe de déforestation la plus importante dans le bassin du Congo. Au Cameroun, la déforestation actuelle est principalement liée à l'agriculture. Le plan d'émergence de ce pays prévoit le développement des infrastructures, la modernisation de l'appareil de production national ainsi que l'exploitation minière. Au Gabon, le taux de déforestation plus faible s'explique par la dynamique agricole et l'ouverture des routes. Le plan d'émergence ambitieux y prévoit la modernisation de l'infrastructure ainsi que le développement de l'agriculture agro-industrielle.

Conclusions. Une mutation des socio-écosystèmes liée à la déforestation est attendue en différents points du bassin du Congo. Les recherches futures devraient maintenant aborder la description de socio-écosystèmes types représentant les différents stades de la transition forestière ainsi que l'identification des facteurs du changement à différentes échelles .

Mots-clés. Déforestation, causes directes, facteurs sous-jacents, Afrique centrale, Cameroun, Gabon.

\section{Drivers of deforestation in the Congo basin tropical forest. A review}

Description of the subject. The forests of the Congo Basin are among the best preserved areas on Earth. Nevertheless, the factors causing deforestation around the world are also present in this subregion. This document presents a literature review of the direct causes and underlying factors of deforestation in the tropical areas and highlights the drivers of deforestation in the Congo Basin and particularly in Cameroon and Gabon.

Literature. Direct causes of deforestation, defined as having a direct cause-and-consequence connection with the destruction of forest cover, are underpinned by economic factors, technological developments, pro-deforestation policy measures and demographic pressures. These factors interact in different ways in the various tropical regions worldwide, which explains the differences and similarities of regional deforestation dynamics. Beside the expansion of infrastructure, the development of mining and timber extraction, agriculture is the main direct cause of deforestation in the Congo Basin. In Cameroon, the current deforestation is primarily driven by agriculture. The State emergency plan includes the development of infrastructure, the modernization of production, equipment and mining. The lack of strategy for rapidly changing demographics might be the most important underlying cause of deforestation. In Gabon, although the deforestation rate is low, agriculture and the opening 
of roads are main drivers. The emergency plan includes modernization of infrastructure and the development of agro-industrial agriculture.

Conclusions. A mutation of socio-ecosystems is expected in different parts of the Congo Basin. Future research should now turn to the analysis of socio-ecosystems representative of the various stages of the forest transition and identify drivers of change at different scales.

Keywords. Deforestation, direct causes, underlying factors, Central Africa, Cameroon, Gabon.

\section{INTRODUCTION}

La déforestation est définie par Kanninen et al. (2007) comme la conversion d'une forêt en une autre forme d'occupation spatiale ou comme la réduction à long terme du couvert forestier sous un seuil de dix pourcents. Un des effets environnementaux les plus importants de la déforestation est l'érosion de la biodiversité. En effet, les forêts tropicales recèlent la plus large diversité spécifique connue (Puig, 2001 ou Teyssèdre, 2004). Ces derniers déclarent que la diminution de la superficie des forêts tropicales ainsi que leur fragmentation seraient responsables de la disparition de $7 \%$ des espèces non exploitées inféodées à ces habitats. Les forêts du bassin du Congo font aujourd'hui partie des zones aux taux de déforestation les plus faibles du globe (de Wasseige et al., 2014). D'après Bellassen et al. (2008), la déforestation représente $0,15 \%$ de la surface forestière $\mathrm{du}$ Bassin du Congo contre $0,51 \%$ en Amérique tropicale ou $0,58 \%$ en Asie tropicale. Actuellement, la perte de la biodiversité est faible dans les forêts du bassin du Congo par rapport aux autres grands massifs forestiers tropicaux (de Wasseige et al., 2014). Pourtant, les processus qui entrainent la destruction du couvert forestier ailleurs dans le monde sont aussi à l'œuvre dans la sous-région. Ces «moteurs de la déforestation » ont été définis comme des éléments ayant un lien cause-conséquence explicite et direct avec la destruction du couvert forestier (Pfaff et al., 2007). On peut citer comme exemples l'ouverture de champs sur la forêt (Kissinger et al., 2012) ou la construction d'infrastructures (Megevand et al., 2013).

Bien souvent, les descriptions de ces liens causaux reposent sur l'utilisation de modèles généralistes et procèdent par simplification de la réalité (Lambin et al., 2001). Ces narrations ont pourtant gagné l'aval du public et influencent les prises de décisions environnementales et l'élaboration de lois (Lambin et al., 2001 ; Geist et al., 2002). Les études antérieures aux années 2000 considèrent souvent des facteurs directs, réputés uniques responsables de la destruction du couvert forestier, comme l'agriculture itinérante (Myers, 1994), l'exploitation minière (Edwards et al., 2014) ou l'ouverture des routes (Théry, 1997). La déforestation peut cependant aussi être corrélée à des facteurs sous-jacents relevant de plusieurs causes comme la pression démographique issue de la combinaison entre le taux de natalité, de mortalité mais aussi des phénomènes de migrations (Mather et al., 1998). La plupart des cas d'étude portent sur l'identification des facteurs responsables de la déforestation à échelle locale. Toutefois, il existe des études comparatives qui tentent de générer une compréhension commune des causes directes et des facteurs sous-jacents à partir de plusieurs études locales (Lambin et al., 2001 ; Geist et al., 2002), ainsi que des études identifiant les causes socio-économiques à l'échelle globale (macro) et leurs répercutions indirectes dans les prises de décisions (Rudel et al., 1997 ; Scrieciu, 2007). Par ailleurs, d'après les projections des impacts des changements globaux sur la biodiversité proposées par Leadley et al. (2010), la conversion de grandes étendues de forêts en une affectation du sol différente (telles que les mines ou des plantations agricoles à grandes échelles) est à prévoir.

La conversion du couvert forestier en un couvert alternatif (agriculture, plantation à grande échelle ou urbanisation) peut être liée à l'augmentation de la pression démographique. La courbe de transition forestière décrite par Mather (1992) montre la relation entre le couvert forestier et le temps. Ce dernier peut être également remplacé par l'augmentation de la densité de population ou un indice du développement économique national (Barbier et al., 2010). Angelsen (2008) divise la courbe de transition forestière en quatre phases :

- le couvert forestier est dense avec un taux de déforestation très faible lié à une densité de population faible ayant un faible impact sur la ressource ;

- la déforestation nette augmente sous l'effet de l'augmentation de la population et de la conversion pour d'autres utilisations de l'espace ;

- la croissance démographique diminue et les révolutions techniques changent la manière de produire, les pressions pour augmenter les surfaces agricoles faiblissent, la courbe atteint son point le plus bas ;

- l'augmentation de la population urbaine change la vision sur la forêt d'une source de bois et de terres agricoles potentielles à une ressource esthétique et récréative. De plus, des plantations d'arbres utiles sur les terres déboisées entrainent l'augmentation nette du couvert forestier. La composition de la forêt nouvellement obtenue est cependant fortement éloignée de la forêt originale. 
Le présent document propose une revue de la littérature des causes directes et des facteurs sousjacents de la déforestation dans les zones tropicales, pour se focaliser ensuite sur les facteurs à l'œuvre dans le bassin du Congo et particulièrement ceux actifs dans deux pays de cette région pris comme exemples afin de déterminer leur situation théorique sur la courbe de transition forestière : le Cameroun et le Gabon. Le Gabon a été choisi pour son couvert forestier important couvrant plus de $88 \%$ du territoire ainsi que pour sa densité de population très faible et majoritairement urbaine (de Wasseige et al., 2014). Des mesures politiques importantes y ont été prises en matière de gestion des forêts obligeant à la rédaction de plans d'aménagement en cas d'exploitation forestière ainsi qu'à la création de 13 parcs nationaux couvrant $11 \%$ du territoire (République du Gabon, 2012; Desclée et al., 2014). Par contraste, le Cameroun a été choisi car il présente la densité de population la plus élevée du bassin du Congo entrainant une demande plus élevée en terres agricoles, en bois d'œuvre et en bois-énergie (Schure et al., 2012) et parce qu'il présente la plus faible proportion de couverture forestière du bassin du Congo (Desclée et al., 2014).

La recherche bibliographique s'est appuyée sur les moteurs de recherche Google Scholar, Scopus et ScienceDirect avec les équations de recherche ainsi que les résultats énoncés dans le tableau 1. L'élimination des articles redondant et s'écartant du sujet ainsi que le choix des articles rassemblant le plus de références bibliographiques ont permis de sélectionner les références figurant dans cette synthèse.

\section{CAUSES DE DÉFORESTATION EN ZONE TROPICALE}

\subsection{Causes directes de déforestation}

Plusieurs causes directes ont été identifiées dans la littérature, elles sont rassemblées par Geist et al. (2002) en trois grands groupes :

- l'expansion de l'agriculture,

- l'extraction du bois,

- l'expansion des infrastructures et de l'exploitation minière.

À ces groupes peuvent être ajoutés des facteurs prédisposant tels que la qualité des sols et les problèmes sociaux. L'expansion de l'agriculture constitue le facteur préponderant, qu'il s'agisse de l'agriculture de rente et de l'élevage en forêt amazonienne ou en Asie du Sud-Est ou de l'agriculture itinérante dans le bassin du Congo (Rudel et al., 2009).
Expansion de l'agriculture. Plusieurs auteurs ont identifié l'agriculture comme étant de loin la principale cause de déforestation dans le monde tropical (Geist et al., 2002 ; Rudel et al., 2009 ; Kissinger et al., 2012). Elle peut être subdivisée en plusieurs types d'activités pouvant toutes entrainer la conversion de forêts :

- l'agriculture familiale à vocation vivrière telle que l'agriculture itinérante sur brûlis,

- les cultures permanentes ou semi-permanentes, qu'elles soient familiales ou agro-industrielles, et l'élevage à grande échelle.

Des différences régionales s'expriment par l'importance relative de ces différents types d'agriculture : l'Amazonie est plus sujette à l'élevage à grande échelle (Tsayem Demaze, 2008 ; Rudel et al., 2009); la forêt tropicale humide africaine serait peu à peu rongée par l'agriculture familiale vivrière et de rente (Hosonuma et al., 2012) ; enfin, dans le sud-est asiatique, la conversion de la forêt en terres arables a d'abord été induite par l'ouverture de routes en forêt et l'allocation de subsides pour la colonisation des forêts denses par les agriculteurs familiaux (Rudel et al., 2009), puis par une politique de promotion des plantations industrielles (De Koninck, 2005).

L'agriculture itinérante sur brûlis constitue un mode de production agricole familiale de subsistance qui répond aux besoins primaires des populations. Après l'abattage et la défriche, des parcelles sont mises en culture pendant quelques années. Le terrain est ensuite laissé en jachère pendant plusieurs années voire plusieurs décennies en fonction de la fertilité de la terre, de la pression des adventices, de la pression foncière et de la disponibilité en force de travail. De Wachter (1997) estime ce système agricole durable tant que la densité de population ne dépasse pas les 30 à 40 habitants $\cdot \mathrm{km}^{-2}$ dans le contexte des essarteurs d'Afrique centrale. Au-delà de ce seuil démographique, les terres et les ressources viennent à manquer, ce qui engendre un raccourcissement des temps de jachère entravant le retour de la forêt ainsi que la restauration de la fertilité du milieu biophysique. Sans innovations

Tableau 1. Équations de recherche et nombre d'articles correspondant par moteur de recherche consulté - Research query and corresponding number of articles for each search engine used.

\begin{tabular}{lccr}
\hline & ScienceDirect & $\begin{array}{l}\text { Google } \\
\text { Scholar }\end{array}$ & Scopus \\
\hline Drivers of deforestation & 4832 & 44700 & 472 \\
«Drivers of deforestation » & 328 & 2700 & 85 \\
«drivers of deforestation & 163 & 1470 & 4 \\
$\begin{array}{lcc}\text { central Africa » } \\
\text { «causes de la déforestation » }\end{array}$ & 6 & 279 & 1 \\
\hline
\end{tabular}


agricoles (apport de fertilisation exogène, lutte contre les adventices améliorée), seule l'ouverture de nouveaux champs sur la forêt garantit alors la production des denrées agricoles (Myers, 1994).

L'agriculture permanente ou semi-permanente, qu'il s'agisse de cultures de rente à échelle familiale ou d'agro-industries, a été principalement mise en place en Amazonie et en Asie du Sud-Est dans les années 1960 à 1970 (Tsayem Demaze, 2008 ; Rudel et al., 2009). À cette époque, la forêt a fait l'objet de projets d'infrastructures. L'ouverture de routes ainsi que l'incitation à la colonisation pionnière ont permis la pénétration du massif forestier, le défrichement de la forêt et l'installation de parcelles agricoles permanentes et cultivées selon des pratiques extensives (Déry, 1996). La production familiale vivrière et de rente ainsi que les pâturages pour l'élevage bovin ont chassé les populations autochtones vers l'intérieur des massifs. En Amazonie brésilienne, les agriculteurs pionniers ont été encouragés par l'État à vendre leurs terres à des propriétaires de ranchs développant des élevages à grande échelle, et à déplacer leurs cultures plus avant dans le massif forestier (Déry, 1996). Ces grands domaines deviennent alors des freins à la pratique de l'agriculture itinérante des agriculteurs familiaux en limitant les espaces disponibles (Cochet, 1993 ; Rudel et al., 2009). Des cultures permanentes ont été mises en place dans toutes les zones tropicales. Les produits issus de ces plantations, qu'il s'agisse de canne à sucre, d'huile de palme, d'hévéa, de coton ou de soja sont destinés principalement au marché agro-industriel ainsi que dans une plus faible mesure à la production d'agro-carburants (Polet, 2011).

Depuis la crise financière et alimentaire de 2007-2008, les acquisitions de grandes surfaces de terres arables dans les pays du Sud sont devenues une préoccupation importante des institutions internationales, de la société civile ainsi que de la presse mondiale (Cotula et al., 2009 ; Karsenty et al., 2012). Ce phénomène de «néocolonialisme agraire » engendre une pression forte sur les espaces forestiers qui est difficile à quantifier. En effet, ces espaces ne sont pas considérés comme des terres arables et leur conversion n'est pas toujours prise en compte.

Extraction du bois. Le bois d'œuvre est surtout exploité de façon dévastatrice en Amérique du Sud et en Asie du Sud-Est (Hosonuma et al., 2012). D'après Fleury (2000), les forêts tropicales auraient été exploitées pour leur bois précieux puis pour leur bois d'œuvre et enfin pour la production de pâte à papier, mais l'exploitation aurait été différente sur les trois continents du fait de la répartition des essences : «En Afrique, on dénombre un grand nombre d'essences avec une densité spécifique faible, les arbres sont donc difficiles à localiser et à extraire. A contrario, en Asie, l'éventail des espèces est plus faible, la forêt est plus homogène et donc plus facile à exploiter. En Amérique latine, l'éventail spécifique semble plus proche de la forêt asiatique mais les forêts sont plus hétérogènes. » (Fleury, 2000).

Le bois de feu est un combustible ligneux brûlé sous forme de bois ou de charbon et fournissant l'énergie nécessaire à la cuisson des aliments et la transformation des produits agricoles (Ozer, 2004). D'après la FAO (2005), 80 à $90 \%$ du bois prélevé en Afrique et en Asie du Sud-Est servirait de combustible pour la préparation des repas ou serait utilisé comme bois de chauffe. D'après Ozer (2004), jusqu'à la grande période de sècheresse des années 1960, le bois de feu était généralement considéré comme une ressource infinie en Afrique sahélienne, l'offre étant bien plus élevée que la demande. Le bois était au Sahel pratiquement la seule source d'énergie utilisée en milieu rural et urbain. La sècheresse des années 1960 a entre autres provoqué un exode rural massif entrainant une concentration de la population dans les milieux urbains ainsi qu'une forte demande en bois-énergie (Benjaminsen, 1996). À l'heure actuelle, le bois et le charbon répondent encore à plus de $90 \%$ des besoins en énergie des populations rurales et urbaines des pays du Sahel, cette valeur ayant peu évolué sur les 50 dernières années (Gazull et al., 2014).

Développement des infrastructures et du secteur minier. Le développement des infrastructures englobe l'urbanisation, les réseaux de transport, de communication, de production et de conduction d'énergie ainsi que le secteur minier.

L'expansion des villes produit un impact direct sur les forêts de par la déforestation périurbaine pour l'expansion de la zone urbaine (Mertens et al., 1997), mais aussi un impact indirect par l'augmentation des besoins en bois de feu et en produits agricoles pour alimenter la population urbaine (Mather et al., 1998 ; Marien, 2009 ; Vermeulen et al., 2011).

Si les impacts directs des infrastructures routières sur le couvert forestier sont assez faibles, les impacts indirects et induits constituent eux une menace importante en modifiant la dynamique économique de la zone nouvellement accessible (Megevand et al., 2013). De nombreuses études rapportent l'impact du développement de routes ou du chemin de fer sur la migration des populations en forêts, la chasse et l'exploitation forestière illégale (Garcia et al., 2014 ; Laurance et al., 2014). L'ouverture de routes en forêt tropicale permet le désenclavement et le transport des produits de l'agriculture et des produits issus de la forêt comme le bois, les fruits, le gibier, mais est à l'origine de perte ou de fragmentation des habitats et d'une dégradation environnementale souvent irréversible (Garcia et al., 2014 ; Laurance et al., 2014). 
En 1970, le programme d'intégration nationale élaboré par le régime brésilien avait pour objet la construction de routes en Amazonie brésilienne afin de désenclaver la zone et de favoriser l'exploitation du territoire pour permettre le développement économique et social et assurer la souveraineté du pays (Demaze, 2008). Théry (1997) décrit l'évolution du réseau routier en Amazonie comme suit : « les routes nationales peuvent être très ramifiées et s'enfoncer sur des dizaines de kilomètres de la route asphaltée, élargissant le couloir de défrichement accessible aux colons et la zone impactée par l'infrastructure routière; ces routes sont ensuite prolongées en pistes érigées par les exploitants forestiers et les agriculteurs pionniers afin d'exploiter de nouvelles zones forestières et de les convertir en terres agricoles. »

En Thailande, le programme de construction de routes a aussi commencé dans les années 1970 afin d'aider l'armée à sécuriser le nord du pays contre les atteintes communistes (Cropper et al., 1999). L'ouverture des routes a permis à une population rurale croissante de s'établir en forêt et d'y développer une agriculture de subsistance.

Les impacts du secteur minier sur le couvert forestier peuvent être de deux types :

- les impacts directs de l'extraction minière qui incluent la pollution ainsi qu'une dégradation de l'habitat naturel par l'élimination du substrat sur des surfaces allant de moins d'un hectare à des dizaines d'hectares en fonction du minerai recherché. Cet impact est irréversible sans action de réhabilitation (Kabulu et al., 2008 ; Edwards et al., 2014) ;

- les impacts indirects qui concernent la construction d'infrastructures de transport et de production d'énergie et l'afflux de population dans des zones jusque-là peu peuplées avec comme conséquences le défrichement pour l'agriculture vivrière, la chasse pour la consommation locale ainsi que l'utilisation de bois d'œuvre et de feu (Brashares et al., 2004 ; Megevand et al., 2013).

\subsection{Facteurs sous-jacents}

Les processus économiques, institutionnels, technologiques ou démographiques caractérisent les contextes régionaux et locaux et expliquent en grande partie les différences observées entre les régions (Mather et al., 1998 ; Geist et al., 2002). Ils étayent les facteurs directs de déforestation et complexifient les relations entre ces facteurs.

Facteurs économiques. D'après Geist et al. (2002), les facteurs économiques seraient les facteurs sous-jacents les plus importants pour expliquer la déforestation des milieux tropicaux.

Plusieurs analyses rassemblées par Ewers (2006) démontrent que la déforestation à une échelle nationale est liée au revenu par habitant suivant une courbe en U inversé. En effet, les revenus nationaux sont dans un premier temps basés sur les revenus issus des ressources environnementales exploitées de manière extractive (chasse, pêche, cueillette, exploitation forestière et minière) ou transformées par l'agriculture. Ces activités reposant sur l'exploitation des ressources naturelles constituent le secteur économique dit «primaire». L'exploitation des ressources a pour conséquence une dégradation environnementale proportionnelle à la croissance économique. Dans un second temps, l'économie nationale n'étant plus basée sur le secteur primaire, les pressions sur l'environnement diminuent dans le pays.

D'après Carr et al. (2005), le facteur économique le plus puissant pour expliquer la déforestation serait la demande, qu'il s'agisse de demandes pour les nécessités de base (fibres vestimentaires, nourriture, bois de construction) ou de demandes plus superficielles (demandes en bois tropicaux ou en fruits exotiques). Ces demandes s'expriment sur des marchés locaux ou globaux et influenceront la conversion de forêts en zones agricoles ainsi que l'intensification des cultures. DeFries et al. (2010) montrent sur base d'images satellites que, à l'échelle nationale, la déforestation est corrélée à la production de produits agricoles destinés à la consommation urbaine ainsi qu'à l'exportation internationale. Selon Geist et al. (2002), la déforestation axée sur les capitaux se réfère aux investissements publics et privés pour développer les frontières politiques, économiques ou pour des raisons sociales. Carr (2004) postule par ailleurs que l'augmentation de la densité de population entraine un raccourcissement des temps de jachère et que seule une intensification de l'agriculture entrainée par l'augmentation de l'économie de marché occasionnerait une diminution de la pression sur le couvert forestier.

Facteurs institutionnels. Il s'agit essentiellement de mesures politiques, prises indépendamment ou par l'approbation d'un plan global, prônant la déforestation comme stratégie de développement (République du Cameroun, 2009) mais aussi de sécurisation des frontières ou de redistribution des terres. On peut citer comme exemples la promotion de la colonisation du terrain ou encore le transport ou les subsides octroyés pour des activités axées sur la terre. Des illustrations de ce type de mesures sont données par Théry (1997) ou Demaze (2008) concernant les décisions politiques visant à la mise en place des routes désenclavant la forêt amazonienne avec pour objectif sa colonisation par des agriculteurs. Le régime foncier (insécurité de la propriété, non-respect des droits coutumiers) et les incuries politiques (corruption ou problèmes de management) sont d'autres moteurs de la déforestation. On peut par exemple citer la colonisation forestière 
permettant la consolidation du pays au Brésil (Demaze, 2008) ou en Indonésie (Levang, 1997) ou encore la politique de conversion des forêts en terres arables afin de promouvoir la croissance économique, la modernisation agricole et l'élévation du niveau de vie (Lambin et al., 2001).

Les facteurs culturels ou socio-politiques sont souvent négligés. Toutefois, ils conditionnent les formations politiques et économiques de par l'attitude et le comportement de la sphère publique peu ou pas concernée par l'environnement forestier. On peut citer l'évolution de la perception publique de la forêt, passant d'une perception utilitaire en termes de terres agricoles potentielles ou de bois de feu à un environnement utile en tant que tel pour les activités récréatives (Colson et al., 2009).

Facteurs technologiques. D'après Carr et al. (2005), les avancées technologiques permettent à la fois de procéder à des prélèvements massifs mais aussi plus sélectifs des ressources. La déforestation liée à l'utilisation d'équipements lourds a entrainé le déclin drastique des forêts asiatiques. En Amérique latine, malgré l'exode rural, les grands propriétaires terriens intensifient de façon considérable leur production. Par contre en Europe, l'exode rural lié à l'avènement des nouvelles technologies agricoles lors de la révolution industrielle a permis une intensification de la production sur les terres non urbanisées les plus adaptées et l'abandon des terres les moins productives à la recolonisation forestière (Mather et al., 1998). Ces avancées technologiques fonctionnent donc à double sens et sont liées aux autres facteurs.

Facteurs démographiques. L'augmentation de la population résultant d'une natalité élevée et d'une baisse de la mortalité peut être suffisante pour entrainer l'expansion de l'espace agricole et une déforestation à l'échelle nationale (Myers, 1994). Dans certains contextes, la croissance naturelle de la population reste faible et seule l'arrivée d'une population exogène peut déclencher un processus de déforestation (Geist et al., 2002). L'exode rural complexifie ces tendances en influençant la main-d'œuvre disponible dans les zones rurales et en concentrant la demande dans les zones péri-urbaines (Rudel et al., 2009 ; DeFries et al., 2010). Ainsi, les facteurs démographiques ont une influence variable sur la déforestation et sont fortement dépendants du contexte (Carr et al., 2005 ; Mena et al., 2006).

\section{BASSIN DU CONGO}

En ce qui concerne le bassin du Congo, la faible pression démographique, la difficulté d'accès et le manque d'infrastructures ont permis de protéger le couvert forestier de cette région du monde pendant une longue période (Megevand et al., 2013). D'après Ernst et al. (2013), le taux annuel de déforestation est passé de $0,13 \%$ dans la période 1990 à 2000 à $0,26 \%$ dans la période 2000 à 2005. Cette augmentation est aussi observée pour la reforestation relatant une relative stabilité du taux de déforestation net dans le bassin du Congo mais aussi la complexité de la dynamique du changement du couvert forestier. Pour illustrer ce qui se passe dans cette région, nous nous pencherons sur deux pays-clés du bassin du Congo : le Cameroun et le Gabon, choisis comme expliqué en préambule pour leur position respective sur la courbe de transition forestière. Tous les pays du bassin du Congo présentent un programme d'émergence économique à des horizons différents. Grâce à ces plans d'émergence, édictés par les gouvernements nationaux, nous tenterons aussi de prédire l'évolution probable de la déforestation dans ces deux pays.

Le Cameroun présente la densité de population la plus élevée des six pays constituant le bassin du Congo $^{1}$ ( 45 habitants $\cdot \mathrm{km}^{-2}$ ) et le taux de croissance de la population y était de $2,7 \%$ en 2008 (Tchatchou et al., 2015). Cette population est majoritairement concentrée dans les centres urbains. L'augmentation de la pression démographique entraine une demande accrue en produits agricoles et en bois de feu prélevés au détriment du couvert forestier.

D'après de Wasseige et al. (2014), la forêt couvrait plus de $88 \%$ du territoire gabonais en 2010. Le taux de déforestation net est de $0,34 \%$ pour la période allant de 1990 à 2000. Elle était principalement liée à l'exploitation forestière et à l'ouverture des routes ainsi qu'à la conversion de terres forestières en cultures, prairies ou savanes. La période 2000-2010 est caractérisée par un taux de déforestation observé de $0,09 \%$ (pas significativement différent de zéro). D'après Ernst et al. (2013), la diminution de la déforestation nette est liée à l'augmentation de la reforestation brute plutôt qu'à la diminution de la déforestation brute. Les principales explications du ralentissement de la déforestation nette sont la combinaison de la faible densité de population, de la faible dynamique agricole et de l'obligation des exploitants forestiers à réaliser des plans d'aménagement ainsi que la création des 13 parcs nationaux en 2002 à la suite du sommet de la terre de Johannesburg. Toutes ces raisons entraineraient une recolonisation par la forêt des zones agricoles abandonnées ainsi que des routes forestières inutilisées (Desclée et al., 2014). Mais il est possible également que la différence entre les deux périodes ne

${ }^{1}$ Qui sont la République Démocratique du Congo, la République Centrafricaine, le Cameroun, la République du Congo, le Gabon et la Guinée Équatoriale. 
soit tout simplement pas significative et que le Gabon constitue donc depuis deux décennies un pays à très faible déforestation.

\subsection{Facteurs directs de la déforestation}

Expansion de l'agriculture. D'après Hosonuma et al. (2012), l'expansion de l'agriculture serait responsable des trois-quarts de la déforestation en Afrique répartie à parts égales entre l'agriculture de subsistance pratiquée pour alimenter le marché local et l'agriculture industrielle. Cette dernière peut être divisée en plantations agro-forestières sous forêt (principalement de cacaoyers et caféiers), considérées comme une cause de dégradation forestière, tandis que les plantations monospécifiques consécutives à une conversion des forêts (principalement hévéa, palmiers à huile, bananier plantain et théier) sont considérées comme des facteurs de déforestation (De Wachter, 1997). En Afrique, le développement de plantations agro-industrielles a commencé sous la colonisation. Lors des indépendances, ces grandes surfaces d'hévéa, de coton ou de canne à sucre ont été le plus souvent nationalisées avant d'être en partie privatisées dans les années 1980 à 1990 (Megevand et al., 2013). Les instabilités politiques et commerciales ainsi que le manque d'infrastructures ont ensuite limité les investissements étrangers engendrant un abandon partiel de ces grandes plantations permettant la recolonisation forestière. Les investissements ont repris dans les années 2000 grâce à une certaine stabilisation politique et au développement des axes routiers et des ports (Megevand et al., 2013).

Dans le bassin du Congo, les investisseurs sont de nos jours issus des anciennes puissances coloniales européennes et de multinationales asiatiques (Feintrenie, 2014).Au total, plus d'1 500000 ha de terres agro-industrielles auraient été concédées dans le bassin du Congo. Des procédures nationales ont été mises en place pour limiter le risque d'accaparement des terres et assurer le respect de normes environnementales et sociales, comme l'exigence d'études d'impact environnemental ou d'enquêtes publiques de vacance des terres avant l'allocation des terres. Néanmoins, l'impact social est peu pris en compte dans ces procédures obligatoires, et en particulier l'accord des populations directement touchées par les projets n'est pas exigé des États (Feintrenie, 2014).

Comme pour l'ensemble de la zone tropicale, les principales causes de la déforestation au Cameroun seraient liées à l'agriculture : l'agriculture itinérante sur brûlis ainsi que l'agriculture de rente (Dkamela, 2011). D'après Mertens et al. (2000), la crise économique de 1986 a provoqué une diminution des subsides alloués à la production cacaoyère et caféière entrainant un désintérêt des producteurs pour ces plantations et une réallocation des forces vives vers l'agriculture itinérante, provoquant de la sorte une déforestation plus importante. Le plan d'émergence prévoit d'augmenter les rendements et les superficies agricoles de $30 \%$. Ceci passera par la promotion des grandes exploitations, l'incitation au regroupement en coopératives agricoles ainsi que l'appui à l'installation des jeunes.

La part du secteur agricole dans l'économie gabonaise est importante, mais subit depuis 2000 une faible et constante diminution au profit de l'exploitation $\mathrm{du}$ pétrole et du bois. Malgré cela et par manque d'amélioration de la technologie et des techniques agricoles, la production agricole se fait au détriment des forêts (Tchatchou et al., 2015).

Extraction du bois. Dans les forêts du bassin du Congo, l'exploitation du bois d'œuvre ne constitue pas une cause de déforestation directe, même si dans certaines zones la phase de récolte du bois d'œuvre précède le changement d'affectation de la zone (Karsenty et al., 2012). La mise en place de l'exploitation du bois d'œuvre dans la région selon des pratiques à faible impact implique le respect d'un plan d'aménagement de la zone exploitée et une exportation très faible de grumes $\left(0,5\right.$ à 2 pieds $\cdot h a^{-1}$, soit 5 à $15 \mathrm{~m}^{3} \cdot$ ha $^{-1}$ suivant une rotation de 25 ans). Doucet et al. (2007) estiment que dans le sud-est du Cameroun, cette technique a permis le maintien du couvert forestier et une dégradation limitée de la forêt. D'après Malhi et al. (2013), cette faible densité d'exploitation combinée à la déficience du réseau routier ainsi qu'à la faible densité de population ne provoquent pas les conditions critiques pour la déforestation.

L'exploitation du bois de feu constitue par contre une menace importante. Malgré une densité de population faible et une ressource naturelle abondante, les prélèvements effectués en bois-énergie dans les pays du bassin du Congo seraient bien plus importants que les prélèvements issus de l'exploitation forestière (FAO, 2005). L'augmentation démographique rapide liée à l'urbanisation non contrôlée et à la dépendance de près de $90 \%$ de la population au bois-énergie entraine une déforestation massive dans des rayons de plus en plus vastes autour de certains centres urbains. Par exemple, en République Démocratique du Congo, l'exploitation de cette source d'énergie est informelle et a lieu dans les reliquats de forêts galeries entourant les villes. Plusieurs projets ont vu le jour afin de mettre en place des plantations d'essences à croissance rapide permettant de produire du charbon et du bois de feu et de diminuer ainsi la pression sur la forêt naturelle (Marien et al., 2013), mais ils restent anecdotiques par rapport à la demande.

$\mathrm{Au}$ Cameroun, malgré la faible influence de l'exploitation forestière industrielle sur le couvert 
forestier (Desclée et al., 2014), cette dernière a évolué en dents de scie allant de 2,3 millions de $\mathrm{m}^{3} \cdot \mathrm{an}^{-1}$ jusqu'à 3,5 millions de $\mathrm{m}^{3}$ au cours des 20 dernières années (de Wasseige et al., 2014). L'évolution de l'exploitation du bois énergie est quant à elle difficile à chiffrer. Schure et al. (2012) estiment toutefois la production de combustible à $79 \%$ de la production de bois rond en 2009, ce qui permet d'alimenter $82,6 \%$ de la population en bois-combustible comme première source d'énergie. Le plan d'émergence prône l'exploitation durable des forêts ainsi que la transformation du bois sur place avant exportation dans la continuité des règles édictées par les sociétés forestières certifiées et les mesures politiques gabonaises (Desclée et al., 2014).

Avant le boom pétrolier des années 1970, l'économie du Gabon reposait essentiellement sur le secteur forestier. Actuellement, le secteur pétrolier représente $46 \%$ du PIB ainsi que $84 \%$ des recettes d'exportations, tandis que le secteur forestier représente environ $2 \%$ du PIB gabonais (Tchatchou et al., 2015).

Développement des infrastructures et du secteur minier. Le bassin du Congo est une des régions les moins bien desservies au monde du fait de la faible pénétrabilité de l'environnement forestier et de la présence de nombreux cours d'eau. Cette absence d'infrastructures convenables a passivement " protégé » les forêts naturelles (Megevand et al., 2013). Les gouvernements voient cependant aujourd'hui comme une nécessité, dans une perspective de croissance économique mais aussi de contrôle stratégique, de développer les infrastructures routières et ferroviaires afin de diminuer les entraves aux marchés qu'ils soient nationaux ou internationaux. En effet, la mauvaise qualité des infrastructures de transport est un obstacle à la croissance économique en augmentant les couts ainsi que les temps de transport. La construction et l'extension de ces réseaux seront accompagnés d'impacts négatifs sur le couvert forestier. Le programme de développement économique du Cameroun (République du Cameroun, 2009) se fonde entre autres sur le développement des infrastructures. $\mathrm{Ce}$ dernier ne prévoit pas l'ouverture de nouvelles routes mais l'amélioration de la qualité du réseau routier existant ainsi que la construction d'un chemin de fer. L'objectif est de désenclaver la plupart des régions du pays où les populations pratiquent actuellement une agriculture de subsistance. L'accessibilité nouvelle des marchés urbains et des voies d'exportation conduira à l'augmentation de l'ouverture de surfaces cultivables sur la forêt. Afin de réduire la dépendance de la population au bois énergie, le gouvernement entend faciliter l'accès à des sources d'énergie alternatives. Ainsi, le plan de développement prévoit la construction de barrages hydroélectriques. La déforestation encourue par la construction de l'ouvrage ainsi que par la superficie inondée pourrait être (au moins en partie) contrebalancée par la réduction de la demande en bois énergie en milieux urbains (Tchatchou et al., 2015). Le Gabon souhaite désenclaver les régions et moderniser les transports, ce qui pourrait entrainer la déforestation d'environ 4000 ha, soit $5 \%$ de la déforestation nette prévue par le plan de développement (Desclée et al., 2014 ; Tchatchou et al., 2015).

Le sous-sol africain renfermerait près de $30 \%$ des ressources minérales mondiales (Taylor et al., 2009). Malgré cette richesse, la production minière africaine représentait moins de $8 \%$ du marché mondial en 2012 (Feintrenie, 2013). D'après cet auteur, les prospections minérales en Afrique centrale ont cependant mis en lumière certaines des plus grandes réserves de fer, de cobalt, de nickel, de chrome mais aussi d'or et de diamants au monde. Une augmentation de la production est prévue en réponse à la demande mondiale en métaux et pierres précieuses. Dans ce contexte, le gouvernement camerounais entend promouvoir l'exploitation minière. L'exploitation minière prévue par certains plans d'émergence est accompagnée de grands projets d'infrastructure comme des routes ou des chemins de fer pour l'évacuation du minerai assortis de barrages hydroélectriques pour l'approvisionnement énergétique des chantiers d'extraction (République du Cameroun, 2009; République du Gabon, 2012). D'après Tchatchou et al. (2015), les permis miniers entrent en compétition avec les surfaces forestières ; l'exploitation minière pourrait ainsi être à l'origine de la destruction directe de plus de 940000 ha de forêt au Cameroun.

\subsection{Facteurs sous-jacents}

Facteurs économiques. L'économie des pays du bassin du Congo repose principalement sur le secteur primaire, qu'il s'agisse de l'exploitation du pétrole au Gabon, en Guinée Équatoriale et en République du Congo ou de l'agriculture, de la foresterie, de la chasse et de la pêche au Cameroun, en République Centrafricaine et en République Démocratique du Congo (African Development Bank Group, 2015). Comme expliqué dans les plans d'émergence de ces pays, la valorisation économique des ressources naturelles est au cœur de la volonté de développement exprimée par les gouvernements (GRIP, 2014). Cette valorisation passe par l'exploitation forestière, minière et agricole afin d'optimiser le développement économique à court terme.

Facteurs institutionnels. Les régulations de la propriété foncière apparues après les indépendances respectives sont ineffectives, les répartitions ayant été 
faites de manière arbitraire sans prendre en compte les droits coutumiers engendrant des conflits importants. L'État est la plupart du temps consacré gestionnaire exclusif des terres (Nguema Ondo Obiang et al., 2011). De plus, la corruption est souvent de mise et permet l'acquisition de grandes surfaces par des sociétés de plantation ou d'exploitation ou encore des élites du pays aux dépens des droits coutumiers des populations locales. Plusieurs exemples ont transparu dans la presse internationale, dont le plus médiatisé reste le projet d'huile de palme de l'entreprise américaine Heracles. Cet investisseur, présentant sa plantation comme une initiative de développement, a pour projet la création d'une plantation de 73000 ha au sud-ouest du Cameroun. La mise en place passerait par l'exploitation de 3 milliards de $\mathrm{m}^{3}$ de bois ainsi que par la violation du droit camerounais en termes d'exploitation illégale et de non-respect des droits coutumiers des populations locales (Oakland Institute et al., 2013).

Facteurs démographiques. Le bassin du Congo présente une densité de population faible variant de 5 à 45 habitants $\cdot \mathrm{km}^{-2}$ en fonction des pays, assortie d'un accroissement annuel de 2 à $4 \%$ (Tchatchou et al., 2015). Cette population majoritairement rurale migre vers les centres urbains à la recherche d'emplois et de conditions de vie plus confortables. Étant donné cette faible densité, seuls les cas d'augmentation brusque de la densité de population par des mouvements migratoires en lien avec des conflits ou l'émergence de sociétés extractives ou agro-industrielles auront un effet significatif sur le couvert forestier (Geist et al., 2002). Toutefois, comme expliqué précédemment, la croissance démographique des centres urbains et l'augmentation de la demande en produits agricoles et bois de feu qui en découle entrainent une déforestation à faible échelle.

Le plan d'émergence camerounais n'annonce pas de stratégie spécifique de l'État concernant l'évolution démographique. Or, si la croissance démographique se poursuit, la population camerounaise doublera entre 2010 et 2035. Comme démontré par DeFries et al. (2010), cette augmentation de la population majoritairement urbaine provoquera une augmentation de la demande en production de vivres et en bois, ainsi que le développement des infrastructures, ce qui aura un impact sur la forêt. Le Gabon, par contre, présente une densité de population très faible $\left(4,8\right.$ habitants $\cdot \mathrm{km}^{-2}$ en 2012) et majoritairement concentrée en zone urbaine, seuls $15 \%$ de la population vivent en zone rurale (de Wasseige et al., 2014).

\section{LA TRANSITION FORESTIÈRE}

D'après les éléments développés dans les paragraphes précédents, nous pouvons maintenant situer nos cas d'étude sur la courbe de transition forestière (Mather, 1992 ; Angelsen, 2008). Le Cameroun, dont l'économie repose sur des activités essentiellement extractrices, se positionne dans la phase décroissante de la courbe de transition forestière sous l'effet combiné des causes directes et des facteurs sous-jacents comme l'augmentation de la densité de population mais aussi la conversion de la forêt pour d'autres affectations des terres (telles que l'agriculture ou les infrastructures). Le Gabon, bien que présentant un taux de déforestation très faible, se situe lui aussi dans la phase descendante de la courbe de transition forestière. Les activités économiques sont principalement liées aux activités extractrices. Notons que dans les perspectives de développement, le gouvernement entend développer le secteur industriel par la valorisation des hydrocarbures, du gaz, du potentiel minier. Cette évolution entrainerait le pays dans une phase de déforestation plus intense.

L'étude de la transition forestière exprime l'évolution de l'écosystème forestier. Toutefois, on peut difficilement dissocier cet écosystème des enjeux sociaux qui lui sont associés. La notion de socio-écosystème défini comme un groupe d'acteurs particulier ayant un impact sur un groupe de ressources particulier et affecté d'un ensemble particulier d'institutions (Janssen et al., 2007) permet d'analyser en même temps l'écosystème forestier et les acteurs qui s'y intéressent. On identifie le point de basculement du socioécosystème forestier comme étant le point critique dans une situation en évolution qui la fait basculer vers un développement irréversible (Repenning et al., 2001). Dans le cas d'un socio-écosystème forestier, il peut s'agir du passage d'une phase d'intense déforestation à une phase de stabilisation du couvert forestier, voire de reboisement.

La modélisation permet d'appréhender une réalité simplifiée et d'élaborer des scénarios d'évolution des socio-écosystèmes. Ainsi, Mosnier et al. (2014) ont modélisé l'évolution du couvert forestier dans le bassin du Congo sur base de scénarios de causes potentielles de la déforestation pour 2030. Le développement et l'amélioration des infrastructures de transport diminueraient les couts de production, ce qui augmenterait la compétitivité du bassin du Congo sur les marchés internationaux. D'autre part, la diminution des couts de production permettrait l'augmentation de la compétitivité des produits locaux sur les marchés nationaux au détriment des produits d'importation. Cette augmentation de la demande combinée à la présence d'infrastructures de transport accroitrait la déforestation nette. Si les plans d'émergence se réalisent bien comme le souhaitent ces pays, cette déforestation aura certainement lieu. Toutefois, il faut noter que la modélisation des scénarios d'émergence n'a pas encore été testée. Notons seulement que ces derniers dépendent fortement de la stabilité géopolitique de la 
sous-région, qui reste à court terme très peu favorable aux investissements.

\section{CONCLUSIONS}

Malgré le taux de déforestation actuellement faible recensé dans le bassin du Congo et plus particulièrement au Cameroun et au Gabon, les politiques d'émergence projetées par les gouvernements de ces pays sont susceptibles d'avoir un impact important sur le taux de couverture forestière. En effet, ces stratégies de développement économique reposent sur la promotion de secteurs économiques primaires, basés sur l'exploitation des ressources naturelles (agriculture, foresterie, extraction minière et pétrolière). Une mutation des socio-écosystèmes liée à la déforestation est donc attendue en certains points du bassin du Congo et particulièrement au Cameroun. Il serait souhaitable que les recherches futures approfondissent l'analyse de socio-écosystèmes types représentant les différents stades de la transition forestière, en particulier dans le bassin du Congo, afin de mieux identifier les facteurs du changement à différentes échelles et leurs interactions. De tels travaux permettront de définir des recommandations quant aux politiques publiques à mettre en œuvre pour préserver les biens et services des écosystèmes forestiers de la région tout en permettant un développement économique durable.

\section{Remerciements}

Nous remercions les co-auteurs pour leur contribution. CoForTips fait partie de l'appel à projets Biodiversa 2012 et est co-financé par ERA-Net Biodiversa, avec les bailleurs de fonds nationaux : ANR (France), BELSPO (Belgique) et FWF (Autriche).

\section{Bibliographie}

African Development Bank Group, 2015. Perspectives économiques en Afrique, http://www. africaneconomicoutlook.org/fr/, (21.04.16).

Angelsen A., 2008. How do we set the reference levels for REDD payments. In: Moving ahead with REDD: issues, options and implications. Bogor, Indonesia: CIFOR, 53-64.

Barbier E., Burgess J. \& Grainger A., 2010. The forest transition: towards a more comprehensive theoretical framework. Land Use Policy, 27(2), 98-107.

Bellassen V., Crassous R., Dietzsch L. \& Schwartzman S., 2008. Réduction des émissions dues à la déforestation et à la dégradation des forêts : quelle contribution de la part des marchés du carbone. Etud. Climat, 14, 43.

Benjaminsen T., 1996. Bois-énergie, déboisement et sécheresse au Sahel: le cas du Gourma malien. Sécheresse, 7(3).
Brashares J. et al., 2004. Bushmeat hunting, wildlife declines, fish supply in west Africa. Science, 306, 11801183.

Carr D., 2004. Proximate population factors and deforestation in tropical agricultural frontiers. Popul. Environ., 25(6).

Carr D., Suter L. \& Barbieri A., 2005. Population dynamics and tropical deforestation: state of the debate and conceptual challenges. Popul. Environ., 27(1), 89113.

Cochet H., 1993. Agriculture sur brûlis, élevage extensif et dégradation de l'environnement en Amérique latine. Tiers-Monde, 34(134), 281-303.

Colson V., Lejeune P. \& Rondeux J., 2009. La fonction récréative de la forêt wallonne: évaluation et pistes de réflexion pour son intégration optimale dans l'aménagement intégré des massifs. Forêt wallonne, 101, 3-17.

Cotula L., Vermeulen S., Leonard R. \& Keeley J., 2009. Land grab of development opportunity? Agricultural investment and international land deals in Africa. London: IIED, IFAD; Roma: FAO.

Cropper M., Griffiths C. \& Mani M., 1999. Roads, population pressures and deforestation in Thailand 1976-1989. Land Econ., 75(1), 58-73.

De Koninck R., 2005. L'Asie du Sud-Est. $2^{e}$ éd. Paris : Armand Colin.

De Wachter P., 1997. Économie et impact de l'agriculture itinérante Badjoué (sud-Cameroun). Civilisations, 44, 62-93.

de Wasseige C. et al., éds, 2014. Les forêts du bassin du Congo : état des forêts 2013. Neufchâteau, Belgique : Weyrich.

DeFries R., Rudel Th., Uriarte M. \& Hansen M., 2010. Deforestation driven by urban population growth and agricultural trade in the twenty-first century. Nat. Geosci., 3(3), 178-181.

Demaze M., 2008. La déforestation en Amazonie brésilienne : une rupture apparente entre développement et environnement. L'Amérique latine. Identités Ruptures, 165-187.

Déry S., 1996. Expansion agricole et déforestation : le modèle sud-est asiatique. Cah. Géogr. Québec, 40(109), 29.

Desclée B. et al., 2014. Évolution du couvert forestier du niveau national au régional et moteurs du changement. In : de Wasseige C. et al., éds. Les forêts du Bassin du Congo : état des forêts 2013. Neufchâteau, Belgique : Weyrich, 21-46.

Dkamela G.P., 2011. Le contexte de la REDD+ au Cameroun: causes, agents et institutions. Bogor, Indonésie : CIFOR.

Doucet J.-L. \& Kouadio Y.L., 2007. Le moabi, une espèce «phare » de l'exploitation forestière en Afrique centrale. Parcs Réserves, 62(2), 25-31.

Edwards D. et al., 2014. Mining and the African environment. Conserv. Lett., 7(3), 302-311. 
Ernst C. et al., 2013. National forest cover change in Congo Basin: deforestation, reforestation, degradation and regeneration for the years 1990, 2000 and 2005. Global Change Biol., 19(4), 1173-1187.

Ewers R., 2006. Interaction effects between economic development and forest cover determine deforestation rates. Global Environ. Change, 16(2), 161-169.

FAO, 2005. Global forest resources assessment. Roma: FAO.

Feintrenie L., 2013. Opportunities responsible land-based investments in Central Africa. In: Annual World Bank conference on land and poverty, April 8-11, 2013, Washington, USA.

Feintrenie L., 2014. Agro-industrial plantations in Central Africa, risks and opportunities. Biodivers. Conserv., 23(6), 1577-1589.

Fleury M.-F., 2000.L'exploitation du bois et la déforestation : exemple du Brésil. Inf. Géogr., 64(1), 58-70.

Garcia C. \& Feintrenie L., 2014. Beyond the mirror: tropical forest fragmentation and its impact on rural livelihoods. In: Kettle C.J. \& Koh L.P. Global forest fragmentation. Wallingford, UK: Cabi, 175-199.

Gazull L. \& Gautier D., 2014. Woodfuel in a global change context. Wiley Interdiscip. Rev. Energy Environ., 4(2), $156-170$

Geist H. \& Lambin E., 2002. Proximate causes and underlying driving forces of tropical deforestation. BioScience, 52(2), 143-150.

Hosonuma N. et al., 2012. An assessment of deforestation and forest degradation drivers in developing countries. Environ. Res. Lett., 7(4).

Janssen M., Anderies J. \& Ostrom E., 2007. Robustness of social-ecological systems to spatial and temporal variability. Soc. Nat. Resour., 20(4), 307-322.

Kabulu D.J. et al., 2008. Analyse de la structure spatiale des forêts au Katanga. Ann. Fac. Sci. Agron., 1(2), 12-18.

Kanninen M. et al., 2007. Do trees grow on money? the implications of deforestation research for policies to promote REDD. Bogor, Indonesia : Cifor.

Karsenty A. \& Ongolo S., 2012. Les terres agricoles et les forêts dans la mondialisation: de la tentation de l'accaparement à la diversification des modèles. Demeter, 12, 99-108.

Kissinger G., Herold M. \& De Sy V., 2012. Drivers of deforestation and forest degradation - A synthesis report for REDD+ Policymakers. Vancouver, Canada: Lexeme consulting.

Lambin E. et al., 2001. The causes of land-use and landcover change: moving beyond the myths. Global Environ. Change, 11(4), 261-269.

Laurance W. et al., 2014. A global strategy for road building. Nature, 513, 229-232.

Leadley P. et al., 2010. Biodiversity scenarios: projections of $21^{\text {st }}$ century change in biodiversity and associated ecosystem services. Montreal, Canada: Secretariat of the Convention on Biological Diversity.
Levang P., 1997. La terre d'en face : la transmigration en Indonésie. Montpellier, France : ORSTOM.

Malhi Y. et al., 2013. African rainforests: past, present and future. Philos. Trans. R. Soc. London Ser. B, 368(1625), 20120312.

Marien J.-N., 2009. Forêts périurbaines et bois énergie: quels enjeux pour l'Afrique centrale ? In : de Wasseige C. et al.,éds. Les forêts du Bassin du Congo : état des forêts 2008. Luxembourg: Office des publications officielles des Communautés européennes, 217-230

Marien J.-N., Dubiez É., Louppe D. \& Larzillière A., 2013. Quand la ville mange la forêt : les défis du bois énergie en Afrique centrale. Versailles, France : Quæ.

Mather A.S., 1992. The forest transition. Area, 24(4), 367379.

Mather A.S., Needle C.L. \& Fairbairn J., 1998. The human drivers of global land cover change: the case of forests. Hydrol. Processes, 12, 1983-1994.

Megevand C. et al., 2013. Deforestation trends in the Congo Basin: reconciling economic growth and forest protection. Washington, DC: The World Bank.

Mena C., Bilsborrow R. \& McClain M., 2006. Socioeconomic drivers of deforestation in the northern Ecuadorian Amazon. Environ. Manage., 37(6), 802-815.

Mertens B. \& Lambin E.F., 1997. Spatial modelling of deforestation in southern Cameroon: spatial disaggregation of diverse deforestation processes. Appl. Geogr., 17(2), 143-162.

Mertens B., Sunderlin W.D., Ndoye O. \& Lambin E.F., 2000. Impact of macroeconomic change on deforestation in South Cameroon: integration of household survey and remotely-sensed data. World Dev., 28(6), 983-999.

Mosnier A. et al., 2014. Modeling impact of development trajectories and a global agreement on reducing emissions from deforestation on Congo Basin forests by 2030. Environ. Resour. Econ., 57(4), 505-525.

Myers N., 1994. Tropical deforestation: rates and patterns. In: Brown K. \& Pearce D.W. The causes of tropical deforestation. London: University College London, 27-40.

Nguema Ondo Obiang S.\& Puepi B.,2011.Laproblématique foncière dans les pays d'Afrique centrale: cas du Cameroun et du Gabon. In: Proceedings of the FIG Working week 2011. Bridging the gap between cultures, 18-22 May 2011, Marrakesh, Morocco.

Oakland Institute \& Greenpeace International, 2013. Huile de palme au Cameroun : le double jeu d'Heracles Farms, http://www.greenpeace.org/france/PageFiles/300718/ Le\%20double\%20 jeu\%20Herakles\%202013 .pdf, (14.09.2015).

Ozer P., 2004. Bois de feu et déboisement au Sahel : mise au point. Sécheresse, 15(3), 243-251.

Pfaff A. et al., 2007. Road investments, spatial spillovers and deforestation in the Brazilian Amazon. J. Reg. Sci., 47(1), 109-123.

Polet F., 2011. Expansion des agrocarburants au Sud: dynamique et impacts. Altern. Sud, 18, 7. 
Puig H., 2001. Diversité spécifique et déforestation : l'exemple des forêts tropicales humides du Mexique. Bois For. Trop., 268(2), 41-55.

Repenning N., Goncalves P. \& Black L., 2001. Past the tipping point: the persistence of firefighting in product development. California Manage. Rev., 43(4), 44.

République du Cameroun, 2009. Cameroun, vision 2035. Yaoundé : Ministère de l'Économie, de la Planification et de l'Aménagement du Territoire

République du Gabon, 2012. Plan stratégique Gabon émergent : vision 2025 et orientations stratégiques 2011 2016, http://www.aninf.ga/telechargements/PLAN\%20 STRATEGIQUE\%20GABON\%20EMERGENT.pdf, (21.04.16).

Rudel T. \& Roper J., 1997. Forest fragmentation in the humid tropics: a cross-national analysis. Singapore $J$. Trop. Geogr., 18(1), 99-109.

Rudel T., Defries R., Asner G.P. \& Laurance W.F., 2009. Changing drivers of deforestation and new opportunities for conservation. Conserv. Biol., 23(6), 1396-1405.

Schure J. et al., 2012. Contribution du bois énergie à la satisfaction des besoins énergétiques des populations d'Afrique centrale: perspectives pour une gestion durable des ressources disponibles. In : de Wasseige C. et al.,éds. Les forêts du Bassin du Congo : état des forêts 2010. Luxembourg : Office des Publications de l'Union européenne, 109-122.

Scrieciu S., 2007. Can economic causes of tropical deforestation be identified at a global level? Ecol. Econ., 62(3-4), 603-612.
Taylor C. et al., 2009. Geology and nonfuel mineral deposits of Africa and the Middle East. Reston, VA, USA: US Geological Surveys.

Tchatchou B., Sonwa D.J., Ifo S. \& Tiani A.M., 2015. Déforestation et dégradation des forêts dans le Bassin du Congo : état des lieux, causes actuelles et perspectives. Bogor, Indonésie : CIFOR.

Teyssèdre A., 2004. Vers une sixième grande crise d'extinctions ? In : Barbault R. \& Chevassus-auLouis B., eds. Biodiversité et changements globaux. Enjeux de société et défis pour la recherche. Paris: Association pour la Diffusion de la Pensée Française (ADPF), 24-36.

Théry H., 1997. Routes et déboisement en Amazonie brésilienne, Rondônia 1974-1996. Mappe Monde Maison Géogr., 97(3), 35-40.

Tsayem Demaze M., 2008. Quand le développement prime sur l'environnement: la déforestation en Amazonie brésilienne. Mondes Dév., 143, 97-116.

Vermeulen C. et al., 2011. Enjeux fonciers, exploitation des ressources naturelles et Forêts des Communautés Locales en périphérie de Kinshasa, RDC. Biotechnol. Agron. Soc. Environ., 15(4), 535-544. 